\title{
Specific Proteins in Nontuberculous Mycobacteria: New Potential Tools
}

\author{
Patricia Orduña, ${ }^{1}$ Antonia I. Castillo-Rodal, ${ }^{1}$ Martha E. Mercado, ${ }^{1}$ \\ Samuel Ponce de León, ${ }^{2}$ and Yolanda López-Vidal ${ }^{2}$ \\ ${ }^{1}$ Programa de Inmunología Molecular Microbiana, Departamento de Microbiología y Parasitología, \\ Facultad de Medicina, Universidad Nacional Autónoma de México, 04510 Mexico City, DF, Mexico \\ ${ }^{2}$ División de Investigación Clínica, Facultad de Medicina, Universidad Nacional Autónoma de México, 04510 Mexico City, DF, Mexico
}

Correspondence should be addressed to Yolanda López-Vidal; lvidal@unam.mx

Received 25 October 2014; Revised 20 December 2014; Accepted 21 December 2014

Academic Editor: Mehdi Mirsaeidi

Copyright (C) 2015 Patricia Orduña et al. This is an open access article distributed under the Creative Commons Attribution License, which permits unrestricted use, distribution, and reproduction in any medium, provided the original work is properly cited.

Nontuberculous mycobacteria (NTM) have been isolated from water, soil, air, food, protozoa, plants, animals, and humans. Although most NTM are saprophytes, approximately one-third of NTM have been associated with human diseases. In this study, we did a comparative proteomic analysis among five NTM strains isolated from several sources. There were different numbers of protein spots from M. gordonae (1,264), M. nonchromogenicum type I (894), M. nonchromogenicum type II (935), M. peregrinum (806), and M. scrofulaceum/Mycobacterium mantenii $(1,486)$ strains, respectively. We identified 141 proteins common to all strains and specific proteins to each NTM strain. A total of 23 proteins were selected for its identification. Two of the common proteins identified (short-chain dehydrogenase/reductase SDR and diguanylate cyclase) did not align with M. tuberculosis complex protein sequences, which suggest that these proteins are found only in the NTM strains. Some of the proteins identified as common to all strains can be used as markers of NTM exposure and for the development of new diagnostic tools. Additionally, the specific proteins to NTM strains identified may represent potential candidates for the diagnosis of diseases caused by these mycobacteria.

\section{Introduction}

Mycobacteria that are not members of the Mycobacterium tuberculosis complex (Mycobacterium africanum, Mycobacterium bovis, Mycobacterium bovis BCG, Mycobacterium canettii, Mycobacterium microti, and Mycobacterium tuberculosis) or leprosy are classified as nontuberculous mycobacteria (NTM). The NTM group comprises more than 150 species that are widely distributed in many different environments (http://www.bacterio.cict.fr/m/mycobacterium.html). NTM have been isolated from water, soil, air, food, protozoa, plants, animals, and humans $[1,2]$. Although most NTM are saprophytes, approximately one-third of NTM have been associated with human diseases $[3,4]$.

NTM infection is relatively uncommon and they are more frequently observed in immunocompromised individuals [5]. However, the rate of disease caused by NTM in individuals without abnormalities that would predispose them to infection appears to be increasing [6]. The development of new epidemiological tools, which are based on molecular techniques, has allowed an increased diagnosis of NTM disease and an increase in the identification of NTM species that are responsible for disease $[4,7]$.

Person to person transmission of NTM has not been reported, and it is generally accepted that NTM infections are acquired from environmental sources (water, soil) and that they are responsible for many nosocomial infections and occupational diseases. Some authors have indicated that NTM infections were directly related to exposure to contaminated water as they demonstrated to isolate the same clones from the water and patients $[1,8,9]$. Nevertheless, there is also a correlation between NTM-contaminated metallic fluids and aerosols with hypersensitive pneumonitis, asthma, and bronchitis observed in metallurgical workers [10]. The NTM species associated with human diseases have been isolated from the lungs, skin, and other soft tissues. Pulmonary infection is the most common disease manifestation and is associated with an increased age of the patient, while the skin 
and soft tissue diseases have not been associated with age or gender $[4,7]$.

Previous exposure to NTM has been proposed as one of the main causes of reduced efficacy of BCG vaccination against pulmonary tuberculosis infection $[11,12]$. Black et al. demonstrated that young adults living in the northern part of Malawi are immunologically reactive to NTM antigens prior to vaccination with BCG [13]. In addition, numerous animal model studies have provided evidence that exposure to NTM before the BCG vaccine application may modulate the immune response that is induced by the BCG vaccine $[14,15]$. Mendoza-Coronel et al. demonstrated that Mycobacterium avium may be implicated in the induction of immune tolerance mechanisms, which could impact the $\mathrm{T}$ cell response that is induced by BCG vaccination [16].

Additionally, exposure to NTM is responsible for the low predictive value of the purified protein derivative (PPD) test. The PPD or the Mantoux reaction is the only available diagnostic tool to identify latent tuberculosis. Ideally, the PPD test could be used as a marker for tuberculosis infection, but unfortunately there is cross-reactivity between NTM infection and BCG vaccination [17-19]. Black et al. described that the IFN-gamma response to $M$. tuberculosis purified protein derivative (PPD) after vaccination was lower in individuals who reacted strongly to NTM antigens [13].

The increase of NTM infections, the variability of BCG vaccine protection, and the lack of a diagnostic tool for Mycobacterium species infection make it necessary to identify novel proteins that can potentially be used in the development of new vaccines and diagnostic tools against tuberculosis infection [20].

In this study, we did a comparative proteomic analysis of five different NTM strains: three were isolated from the pump water in Mexico City (Mycobacterium gordonae, Mycobacterium nonchromogenicum type II, and Mycobacterium peregrinum), one was isolated from human pulmonary infection (Mycobacterium scrofulaceum/Mycobacterium mantenii), and one was purchased ( $M$. nonchromogenicum type I, ATCC 1953). We identified proteins common to all strains and specific proteins to each NTM strain. Some of the proteins that were common between the strains could be used as markers of NTM exposure.

\section{Materials and Methods}

2.1. Bacterial Strains. Five strains of NTM were used in this study: three were isolated from the pump water in Mexico City as previously described by Castillo-Rodal et al. (M. gordonae, $M$. nonchromogenicum type II, and M. peregrinum) [2], one was isolated from human pulmonary infection (M. scrofulaceum/M. mantenii), and one was purchased ( $M$. nonchromogenicum type II, ATCC 1953) (Table 1). To ensure that all NTM strains were in the metabolically activated state, growth curves of each strain in Sauton medium were determined (Figure 1). All strains were grown to the midlogarithmic phase (10 days for M. nonchromogenicum type I, 20 days for $M$. nonchromogenicum type II, 11 days for M. gordonae, 8 days for $M$. peregrinum, and 17 days for $M$. scrofulaceum $/ M$. mantenii) at $37^{\circ} \mathrm{C}$ with shaking and then

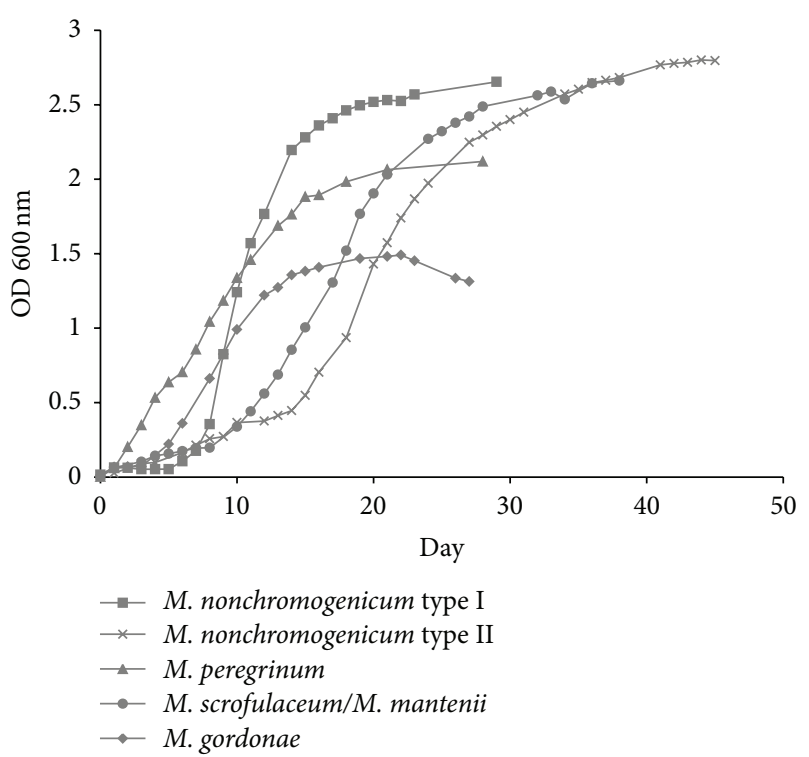

FIGURE 1: Representative growth curves of NTM strains included in this study. Growth curves were realised on Sauton medium at $37^{\circ} \mathrm{C}$ by duplicate.

were harvested by centrifugation, washed three times, and suspended in sterile deionised water.

2.2. Sample Preparation and 2D-PAGE. Cellular proteins were obtained by sonicating the bacteria (Ultrasonic Processor, Cole Parmer Corporation, USA) in the presence of protease inhibitors (10 mM PMSF, $1 \mathrm{mM}$ EDTA; cycles: $1 \mathrm{~min} \mathrm{ON} / 1 \mathrm{~min} \mathrm{OFF}$ ) at $4^{\circ} \mathrm{C}$. For $2 \mathrm{D}-\mathrm{PAGE}$, approximately $80 \mu \mathrm{g}$ of protein for the analytical gels or $100-150 \mu \mathrm{g}$ of protein for the preparative gels was solubilised, denatured, and reduced in sample buffer (4\% CHAPS, $9 \mathrm{M}$ urea, $70 \mathrm{mM}$ 1-dithiothreitol (DTT), $0.001 \%$ bromophenol blue, and $0.1 \%$ 3-10 ampholyte) and was used to rehydrate 11-cm, $\mathrm{pH} 4-7$ IPG strips (ReadyStripTM, IPG strips, Bio-Rad, USA). IEF was carried out on a Multiphor II (Amersham Biosciences, UK) until $52,000 \mathrm{VH}$ at $17^{\circ} \mathrm{C}$. Prior to separation in the second dimension, IPG strips were equilibrated in a solution containing $6 \mathrm{M}$ urea, $30 \%$ (v/v) glycerol, $50 \mathrm{mM}$ Tris-base $\mathrm{pH}$ 8.8 , and $2 \%(\mathrm{w} / \mathrm{v})$ SDS. The strips were equilibrated first for $15 \mathrm{~min}$ with $70 \mathrm{mM}$ DTT and then for $15 \mathrm{~min}$ with $120 \mathrm{mM}$ iodoacetamide. The second-dimension electrophoresis was performed using a $12.5 \%$ polyacrylamide gel (Hoefer SE-600, Amersham Biosciences, UK) with a voltage gradient of 50$150 \mathrm{~V}$ for approximately four hours. Once fixed, the proteins were silver-stained and the gel images were then captured in a digital format for analysis (Molecular Imager GS-800TM Calibrated Densitometer, Bio-Rad, USA).

2.3. Gel Analysis and Spot Selection. 2D-PAGE was performed twice for each strain, and independent cultures were utilised to eliminate technical variation. Gel analysis was performed using PDQuest-Advanced 2D Analysis V8.0 (BioRad, USA). A master image gel (MIG) was integrated with the two duplicate gels of each strain and was utilised for comparison. To estimate and overcome technical variations 
TABLE 1: Description of the NTM strains used in this study.

\begin{tabular}{lcc}
\hline Strain & Source & Growth \\
\hline M. gordonae & Superficial water & Slow \\
M. nonchromogenicum type I (ATCC 19530) & Soil & Slow \\
$\begin{array}{ll}\text { M. nonchromogenicum type II } \\
\text { M. peregrinum }\end{array}$ & Superficial water & Slow \\
M. scrofulaceum/M. mantenii & Water distribution system Mexico City & Fast \\
\hline
\end{tabular}

TABLE 2: Spots identified in the 2D-PAGE gels of cellular proteins from various NTM strains.

\begin{tabular}{lcc}
\hline Strain & Total spots in master image gel & CV $(\%)^{*}$ \\
\hline M. gordonae & 1,264 & 0.2 \\
M. nonchromogenicum type I & 894 & 5.5 \\
M. nonchromogenicum type II & 935 & 0.07 \\
M. peregrinum & 806 & 0.02 \\
M. scrofulaceum/M. mantenii & 1,486 & 0.01 \\
\hline
\end{tabular}

${ }^{*}$ Coefficient of variation: data were normalised according to the total density of the gel image.

between replicates, the spots were quantified for all of the gels. The variation in the coefficients was calculated using a previously described method [21,22]. The spot intensity values were normalised to the total pixel count for each gel. Ten common spots for all strains, 7 specific spots for $M$. gordonae strain, and 2 specific spots for $M$. arupense, $M$. nonchromogenicum, and $M$. peregrinum strains were selected and identified by mass spectrometry. Spot selection criteria included the following: the spot was well defined, the spot had a high intensity, and the spot locations were diversely spaced throughout the gel.

2.4. Protein Identification. The selected spots were identified by a previously described protocol $[21,22]$. Protein identification was performed using a 3200 QTRAP hybrid tandem mass spectrometer (3200 QTRAP, Applied Biosystems, USA) equipped with a nanoelectrospray ion source (NanoSpray II) and a MicroIonSpray II head. Proteins were identified based on their MS/MS spectra datasets using the MASCOT search algorithm (Version 1.6b9, Matrix Science, London, UK). A BLAST search was conducted comparing the sequences to the M. tuberculosis complex and Eubacteria kingdom sequences of the National Center for Biotechnology Information (NCBI) nonredundant database (NCBI nr20070623).

\section{Results and Discussion}

All of the NTM strains that were used in this study were slow growth strains, except for $M$. peregrinum that was a fast growth strain. We determined that the proteins did not have evidence of degradation by a polyacrylamide gel electrophoresis (data not shown).

The cell fractions from the five NTM strains in the mid-logarithmic phase were isolated and then analysed by two-dimensional polyacrylamide gel electrophoresis (2DPAGE). We identify different number of protein spots from M. gordonae (1,264), M. nonchromogenicum type I (894), M. nonchromogenicum type II (935), M. peregrinum (806), and $M$. scrofulaceum/M. mantenii $(1,486)$ strains, respectively
(Figure 2, Table 2). The distribution of the proteins by MM and pI was similar between the five NTM strains analysed (Figure 3).

Comparison of the protein profiles showed that 141 proteins were present in all NTM strains studied and approximately $80 \%$ of the proteins were shared between two or more strains (named common proteins). We also identified proteins present in only one NTM strain (named specific proteins). M. gordonae was observed to have the highest percentage of specific proteins, with $24 \%$ (Table 3 ).

A total of 23 proteins of the five NTM strains studied were selected for their identification by MS-based technologies. Spot selection criteria included the following: the spot was well defined, the spot had a high intensity, and the spot locations were diversely spaced throughout the gel.

We identify ten common proteins to all NTM strains studied (Table 4). Four of these proteins corresponded to informational pathways (RNA polymerase beta subunit, 50S ribosomal protein L7/L12, diguanylate cyclase, and DNA polymerase III), three were related to intermediary metabolism and respiration (adenylate kinase, probable aldehyde dehydrogenase, and enolase), two were identified as conserved hypothetical proteins (WAG31, Rv3075c), and one was related to lipid metabolism (short-chain dehydrogenase/reductase). The 50S ribosomal protein L7/L12, adenylate kinase, enolase, and two hypothetical proteins (WAG31 and $\mathrm{Rv} 3075 \mathrm{c}$ ) have been previously identified in the proteome of M. tuberculosis and M. bovis BCG showing that these proteins are shared with $M$. tuberculosis complex species [23-25]. Interestingly, the ribosomal protein L7/L12 and hypothetical protein WAG31 have been found in the proteome of PPD $M$. tuberculosis and/or PPD M. bovis [26]. Furthermore, the 50S ribosomal protein L7/L12 was described as an immunogenic protein in the BCG Mexico strain suggesting that this protein may be part of the cross-reaction observed between BCG vaccination and NTM exposure [27]. The five remaining proteins were identified for the first time in a mycobacterial proteome in this study. 


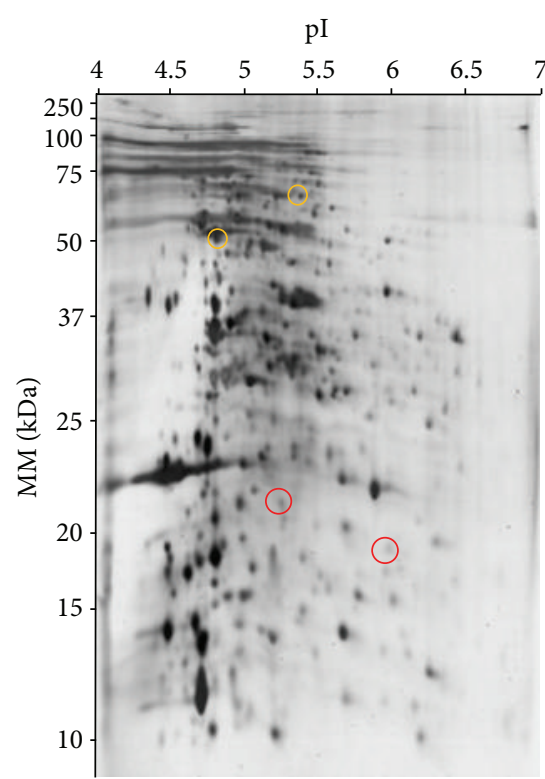

(a)

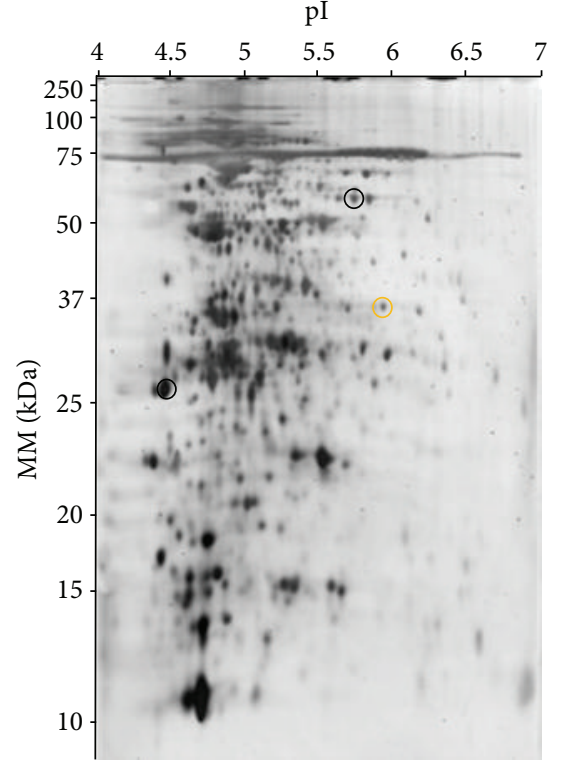

(b)

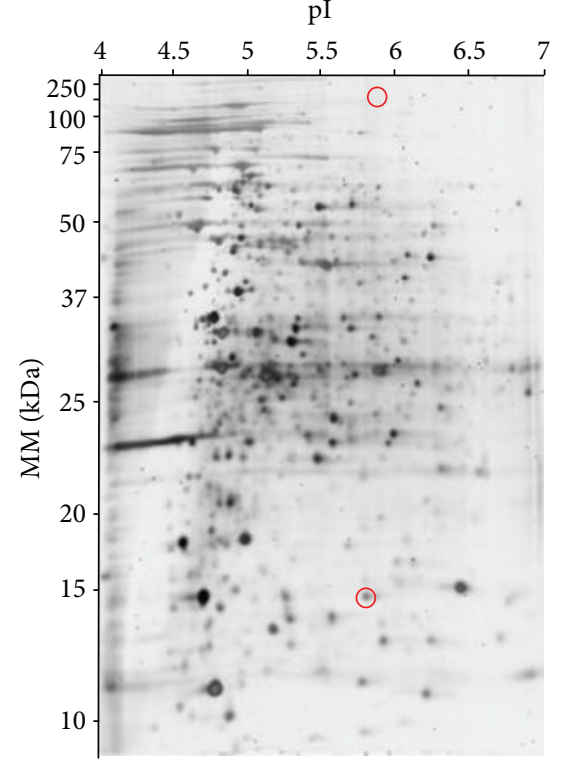

(c)

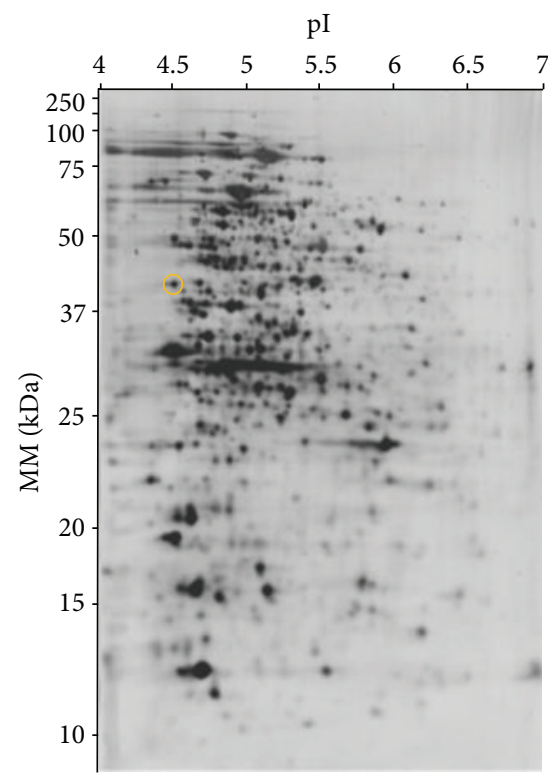

(d)

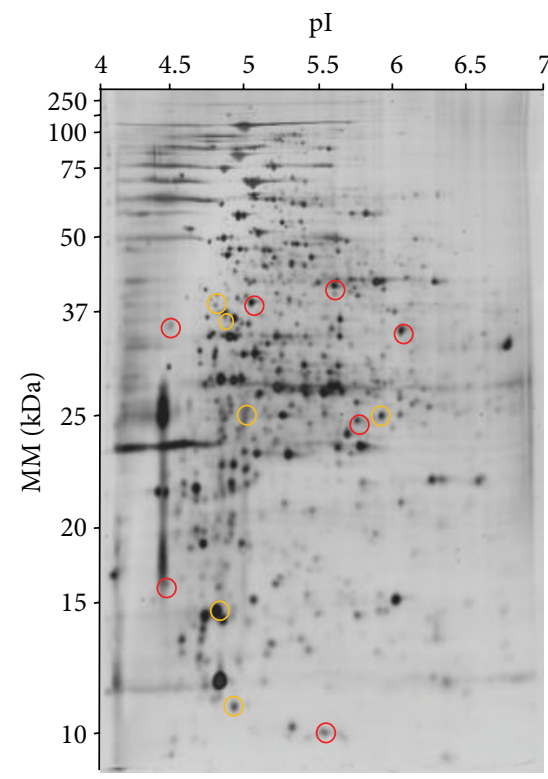

(e)

FIGURE 2: Representative 2D-PAGE of NTM cellular proteins of (a) M. nonchromogenicum type I, (b) M. peregrinum, (c) M. nonchromogenicum type II, (d) M. scrofulaceum/M. mantenii, and (e) M. gordonae. Eighty micrograms of cell proteins was isoelectrically focused in IPG strips ( $\mathrm{pH} \mathrm{4-7)}$ ) and run on sodium dodecyl sulphate (SDS) 12.5\% polyacrylamide gel. Gels were silver-stained and analysed with PDQuest 2D Analysis V8.0 (Bio-Rad, USA). The yellow and red circles were used to identify common proteins to all NTM strains and specific proteins to each NTM strain, respectively, by MS-based techniques.

The proteins of NTM that were identified in this study and have been previously described in the M. bovis BCG proteome can explain the cross-reactivity observed between BCG vaccination and NTM exposition. For example, the 50S ribosomal protein L7/L12, which we determined to be present in all proteomes of NTM strains studied, has been previously described as an immunogenic protein that upregulated the expression of the mannose receptor, CD80, CD86, and MHC class II molecules and it is associated with mycobacterial virulence $[28,29]$.

Moreover, the proteins that have previously been identified in the PPD M. tuberculosis proteome and that were identified in the NTM proteome, such as 50S ribosomal protein L7/L12, adenylate kinase, and hypothetical protein WAG31, may be the cause of the low predictive value of the PPD test to diagnose $M$. tuberculosis infection [26]. In fact, 


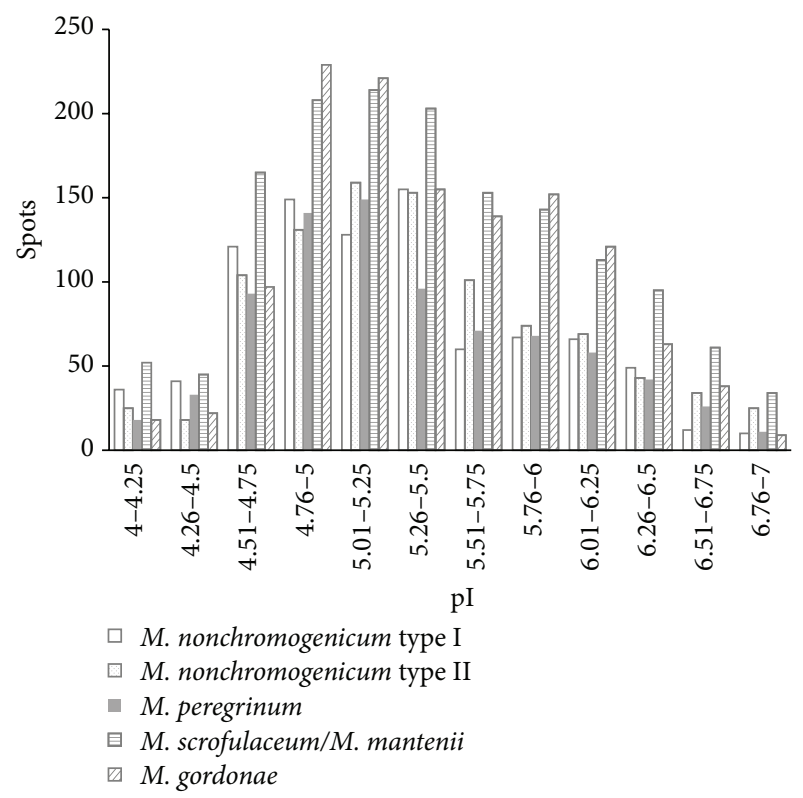

(a)

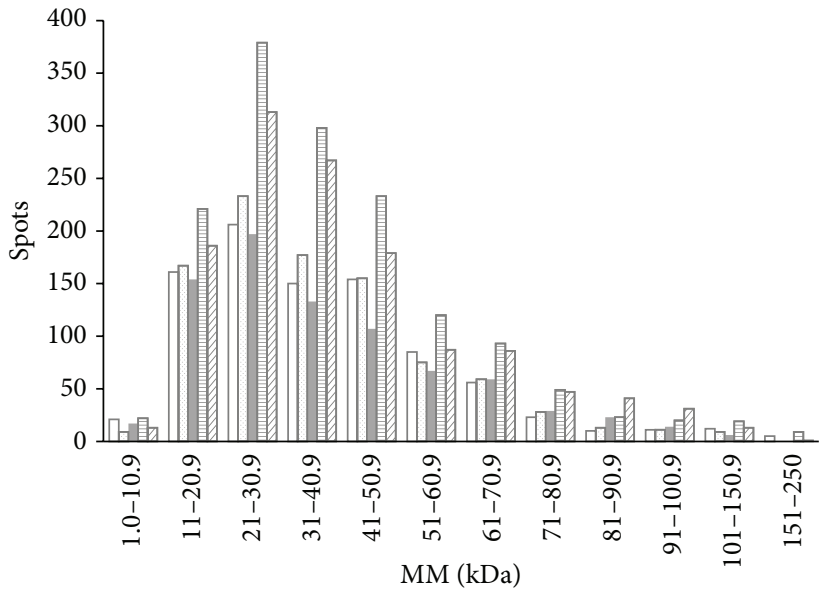

$\square$ M. nonchromogenicum type I

$\square$ M. nonchromogenicum type II

- M. peregrinum

目 M. scrofulaceum/M. mantenii

$\square$ M. gordonae

FiguRE 3: Distribution of proteins by isoelectric point (a) and molecular mass (b). Gel analysis was performed using PDQuest-Advanced 2D Analysis V8.0 (Bio-Rad, USA).

TABLE 3: Common and specific proteins in the NTM strains studied.

\begin{tabular}{lcc}
\hline Strain & Common proteins $^{*}(\%)$ & Specific proteins ${ }^{* *}(\%)$ \\
\hline M. gordonae & $963(76)$ & $301(24)$ \\
M. nonchromogenicum type I & $701(81)$ & $166(19)$ \\
M. nonchromogenicum type II & $723(81)$ & $171(19)$ \\
M. peregrinum & $671(83)$ & $135(17)$ \\
M. scrofulaceum/M. mantenii & $1,231(83)$ & $255(17)$ \\
Proteins common to all strains & $\mathbf{1 4 1}$ &
\end{tabular}

${ }^{*}$ Common proteins were defined as proteins that were present in at least two NTM strains.

${ }^{* *}$ Specific proteins were defined as proteins present only in one NTM strain.

the 50 S ribosomal protein $\mathrm{L} 7 / \mathrm{L} 12$ is an immunogenic protein that induces a strong delayed-type hypersensitivity reaction [28], while the hypothetical protein WAG31 is involved in peptidoglycan synthesis and it has an important role in wall synthesis, cell growth, and cell division of mycobacteria [30].

Thirteen specific proteins from the NTM strains were identified by MS-based technologies (Table 4). We identified two specific proteins to $M$. arupense (deoxyuridine $5^{\prime}$ triphosphate nucleotidohydrolase and probable 3-hydroxylthioester dehydratase), M. nonchromogenicum (conserved hypothetical protein, catalase-peroxidase), and M. peregrinum (mannose-binding lectin, inositol-5-monophosphate dehydrogenase) and seven specific proteins to $M$. gordonae (probable cold shock protein A, putative mannose-specific lectin precursor, superoxide dismutase, malate dehydrogenase, F420-dependent glucose-6-phosphate dehydrogenase, luciferase-like protein, and hypothetical protein SKA58_12772). Four proteins identified as specific to $M$. gordonae (probable cold shock protein A, superoxide dismutase, malate dehydrogenase, and F420-dependent glucose-6phosphate dehydrogenase) have also been identified in the proteomes of $M$. tuberculosis and/or M. bovis BCG [2325]. Moreover, the F420-dependent glucose-6-phosphate dehydrogenase and superoxide dismutase proteins have been identified in PPD $M$. avium and $M$. immunogenum proteomes, respectively $[26,31]$. Interestingly, Dong et al. described that the superoxide dismutase has one immunodominant epitope for cytotoxic T lymphocytes, which are implicated in protective immunity against tuberculosis [32]. These findings suggest that these proteins, which were identified as specific to M. gordonae, are shared with other NTM strains and these may be the cause of cross-reactivity against M. tuberculosis, M. bovis BCG, and/or other NTM.

On the other hand, we identified three specific proteins to $M$. gordonae (putative mannose-specific lectin precursor, luciferase-like protein, and hypothetical protein SKA58_12772) and one specific protein to $M$. peregrinum (mannose-binding lectin) that have not been previously described in any proteomic analysis of mycobacterial strains and did not align with any protein sequences in the $M$. tuberculosis complex database (data not shown). The function of these proteins is not well defined; however, we have known 


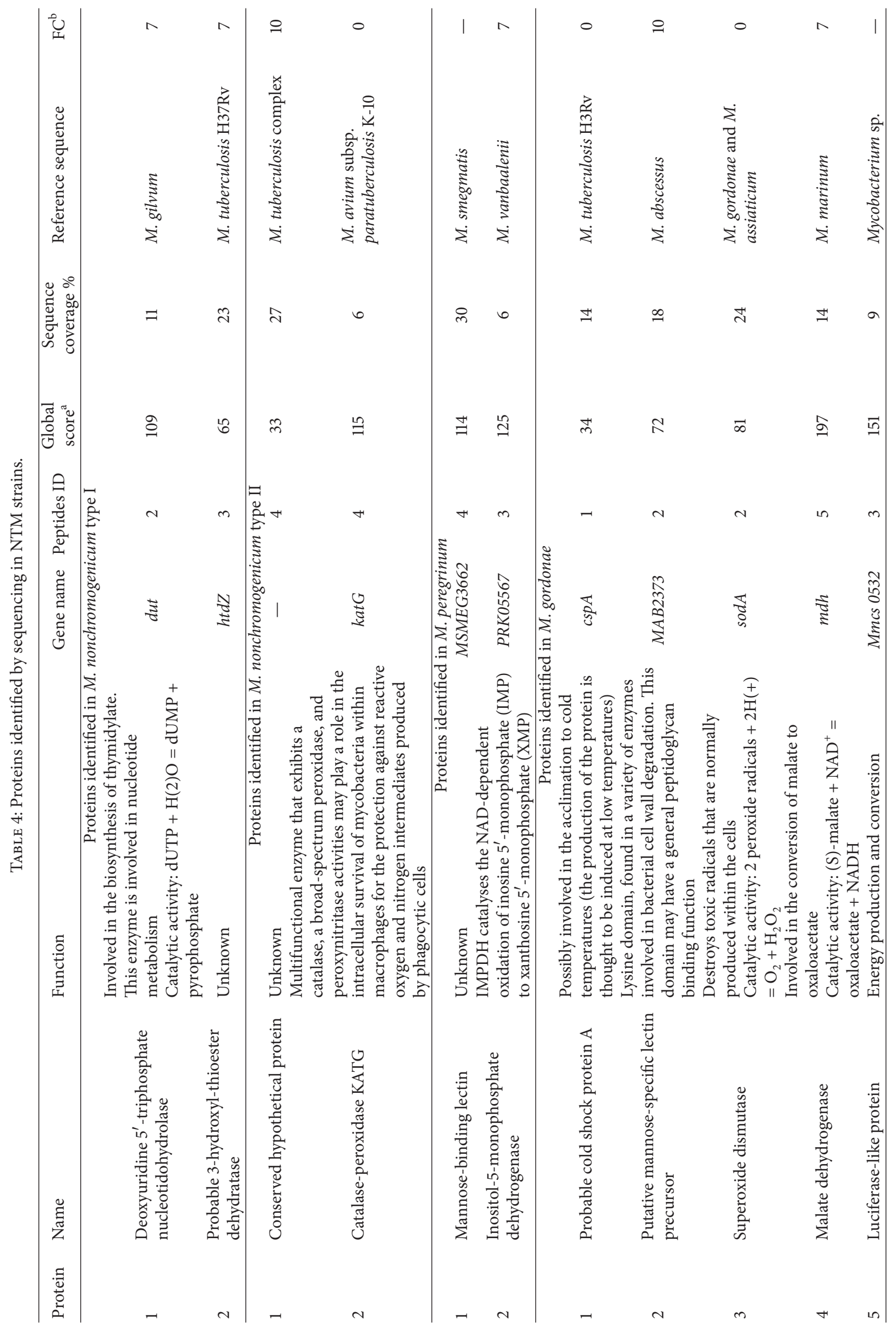




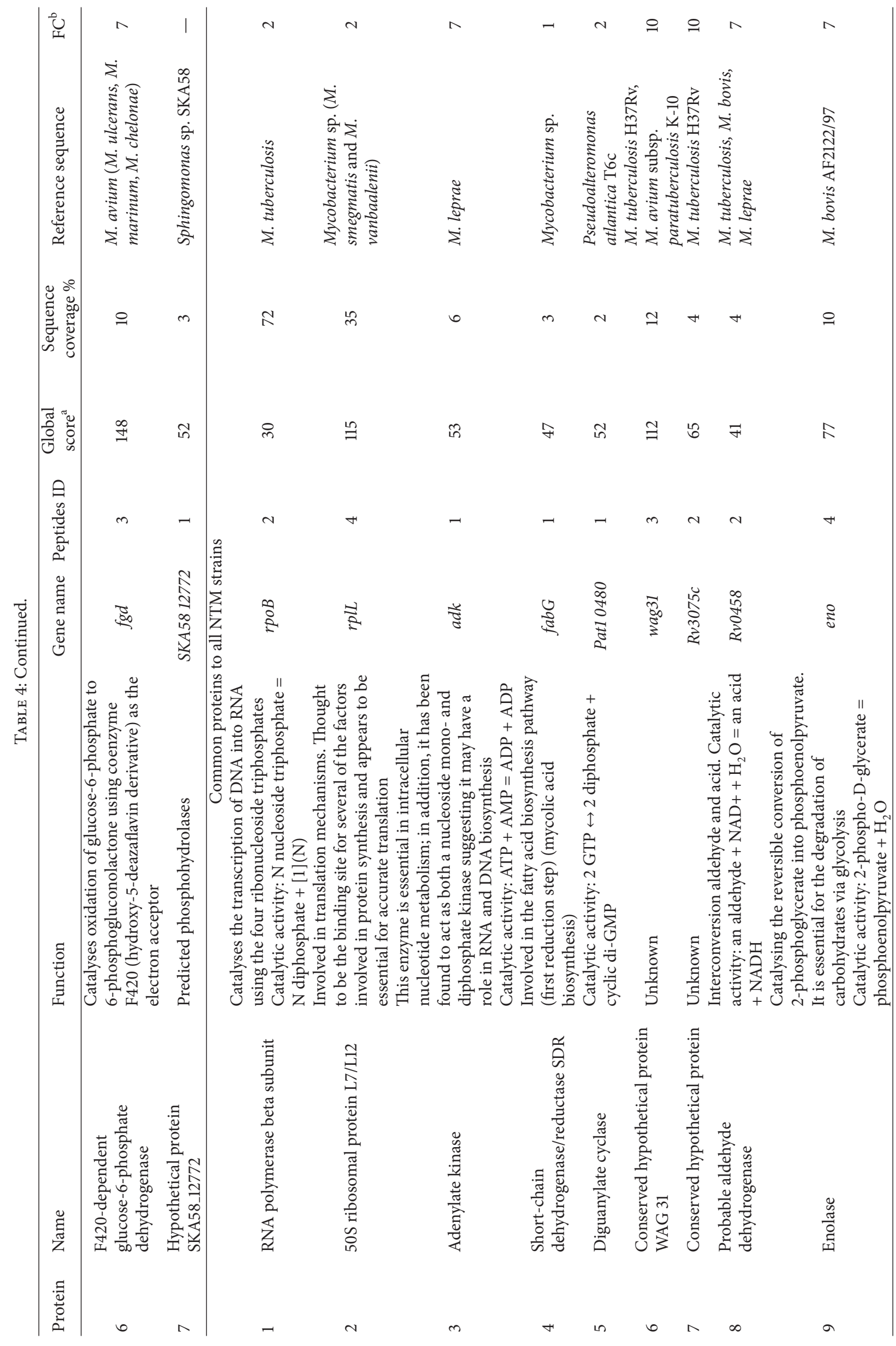




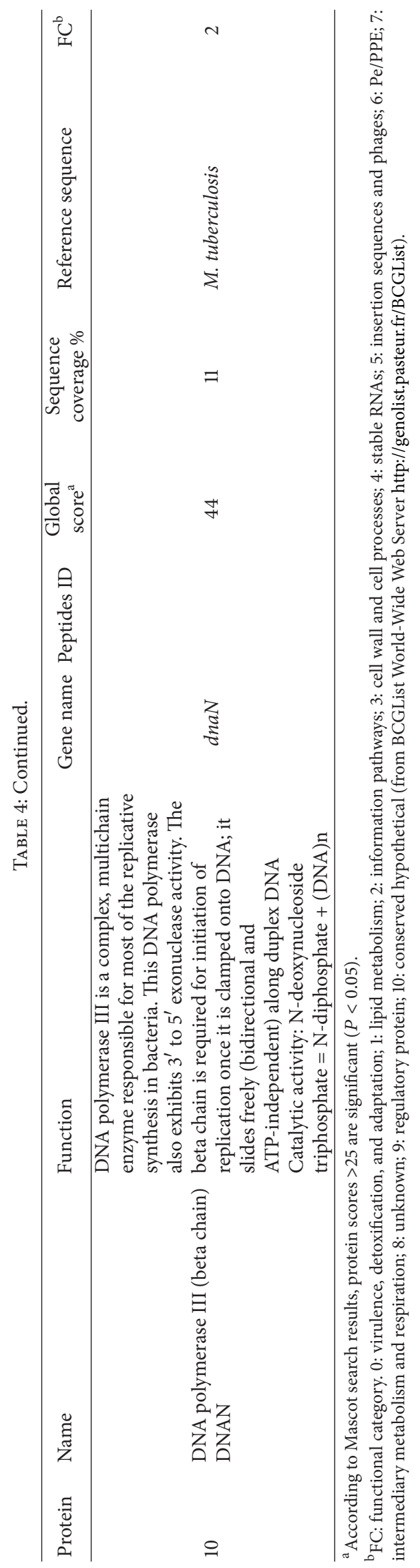


that the lectins can play a major role in the interaction with human cells $[33,34]$. These proteins could be utilised to discriminate between NTM and Mycobacterium tuberculosis complex infections. However, the presence of these proteins in other NTM species that were not included in this study must be determined by specific assays.

\section{Conclusions}

NTM are a group of environmental bacteria that are considered to be potentially pathogenic both to immunocompetent and immunocompromised individuals. Exposure to these bacteria is a factor involved in the variability of the protective efficacy of the BCG vaccine because of cross-reactive antigens that are common between NTM and strains of the $M$. tuberculosis complex. Additionally, exposure to NTM is responsible for the low predictive value of the PPD test due to crossreactivity with similar antigens.

In this study, we described the protein profiles of five NTM strains. Analysis of these profiles indicated the presence of proteins that were both common to and specific to each NTM strain. The common proteins can be utilised as markers of prior exposure to NTM. They can potentially provide a more specific diagnosis with a decreased number of false positives. Also, the proteins that were identified in the proteome of the NTM strains studied can be useful in the development of new diagnostic tools and may help explain the cross-reactivity between the PPD test and the BCG vaccination as described above.

\section{Conflict of Interests}

The authors declare that there is no conflict of interests regarding the publication of this paper.

\section{Acknowledgment}

This work was supported by Consejo Nacional de Ciencia y Tecnología (CONACyT), México, Grant SALUD-2007-C0168729, CONACYT SALUD-2010-C01-140998 and DGAPAIN214713.

\section{References}

[1] J. O. Falkinham III, "Surrounded by mycobacteria: nontuberculous mycobacteria in the human environment," Journal of Applied Microbiology, vol. 107, no. 2, pp. 356-367, 2009.

[2] A. I. Castillo-Rodal, M. Mazari-Hiriart, L. T. Lloret-Sánchez, B. Sachman-Ruiz, P. Vinuesa, and Y. López-Vidal, "Potentially pathogenic nontuberculous mycobacteria found in aquatic systems. Analysis from a reclaimed water and water distribution system in Mexico City," European Journal of Clinical Microbiology and Infectious Diseases, vol. 31, no. 5, pp. 683-694, 2012.

[3] R. M. Thomson, "Changing epidemiology of pulmonary nontuberculous mycobacteria infections," Emerging Infectious Diseases, vol. 16, no. 10, pp. 1576-1583, 2010.

[4] P. M. Cassidy, K. Hedberg, A. Saulson, E. McNelly, and K. L. Winthrop, "Nontuberculous mycobacterial disease prevalence and risk factors: a changing epidemiology," Clinical Infectious Diseases, vol. 49, no. 12, pp. e124-e129, 2009.
[5] B. M. Knoll, "Update on nontuberculous mycobacterial infections in solid organ and hematopoietic stem cell transplant recipients," Current Infectious Disease Reports, vol. 16, no. 9, article 421, 2014.

[6] E. E. Bodle, J. A. Cunningham, P. Della-Latta, N. W. Schluger, and L. Saiman, "Epidemiology of nontuberculous mycobacteria in patients without HIV infection, New York City," Emerging Infectious Diseases, vol. 14, no. 3, pp. 390-396, 2008.

[7] I. M. Orme and D. J. Ordway, "Host response to nontuberculous mycobacterial infections of current clinical importance," Infection and Immunity, vol. 82, no. 9, pp. 3516-3522, 2014.

[8] J. Esteban, N. Z. Martín-de-Hijas, A.-I. Fernandez, R. Fernandez-Roblas, and I. Gadea, "Epidemiology of infections due to nonpigmented rapidly growing mycobacteria diagnosed in an urban area," European Journal of Clinical Microbiology \& Infectious Diseases, vol. 27, no. 10, pp. 951-957, 2008.

[9] M. J. M. Vaerewijck, G. Huys, J. C. Palomino, J. Swings, and F. Portaels, "Mycobacteria in drinking water distribution systems: ecology and significance for human health," FEMS Microbiology Reviews, vol. 29, no. 5, pp. 911-934, 2005.

[10] B. G. Shelton, W. D. Flanders, and G. K. Morris, "Mycobacterium sp. as a possible cause of hypersensitivity pneumonitis in machine workers," Emerging Infectious Diseases, vol. 5, no. 2, pp. 270-273, 1999.

[11] WHO, "BCG vaccine," Weekly Epidemiological Record 4, 2004.

[12] P. Andersen and T. M. Doherty, "The success and failure of BCG-implications for a novel tuberculosis vaccine," Nature Reviews Microbiology, vol. 3, no. 8, pp. 656-662, 2005.

[13] G. F. Black, H. M. Dockrell, A. C. Crampin et al., "Patterns and implications of naturally acquired immune responses to environmental and tuberculous mycobacterial antigens in Northern Malawi," Journal of Infectious Diseases, vol. 184, no. 3, pp. 322-329, 2001.

[14] C. Demangel, T. Garnier, I. Rosenkrands, and S. T. Cole, "Differential effects of prior exposure to environmental mycobacteria on vaccination with Mycobacterium bovis BCG or a recombinant BCG strain expressing RD1 antigens," Infection and Immunity, vol. 73, no. 4, pp. 2190-2196, 2005.

[15] L. Brandt, J. F. Cunha, A. W. Olsen et al., "Failure of the Mycobacterium bovis BCG vaccine: some species of environmental mycobacteria block multiplication of BCG and induction of protective immunity to tuberculosis," Infection and Immunity, vol. 70, no. 2, pp. 672-678, 2002.

[16] E. Mendoza-Coronel, R. Camacho-Sandoval, L. C. Bonifaz, and Y. López-Vidal, "PD-L2 induction on dendritic cells exposed to Mycobacterium avium downregulates BCG-specific T cell response," Tuberculosis, vol. 91, no. 1, pp. 36-46, 2011.

[17] L. Kröger, M. Korppi, J. Pelkonen, M. Pietikäinen, and M. L. Katila, "Development of tuberculin reactivity and sensitization to M. scrofulaceum and M. fortuitum in children BCGvaccinated at birth," European Respiratory Journal, vol. 15, no. 2, pp. 382-387, 2000.

[18] B. L. Marmorstein and D. J. Scheinhorn, "The role of nontuberculous mycobacterial skin test antigens in the diagnosis of mycobacterial infections," Chest, vol. 67, no. 3, pp. 320-324, 1975.

[19] R. E. Huebner, M. F. Schein, G. M. Cauthen et al., "Evaluation of the clinical usefulness of mycobacterial skin test antigens in adults with pulmonary mycobacterioses," The American Review of Respiratory Disease, vol. 145, no. 5, pp. 1160-1166, 1992.

[20] M. Pai, "Alternatives to the tuberculin skin test: Interferon- $\gamma$ assays in the diagnosis of Mycobacterium tuberculosis infection," 
Indian Journal of Medical Microbiology, vol. 23, no. 3, pp. 151158, 2005.

[21] M. Rodríguez-Alvarez, G. Mendoza-Hernández, S. Encarnación, J. J. Calva, and Y. López-Vidal, "Phenotypic differences between BCG vaccines at the proteome level," Tuberculosis, vol. 89, no. 2, pp. 126-135, 2009.

[22] M. Rodríguez-Alvarez, I. D. Palomec-Nava, G. MendozaHernández, and Y. López-Vidal, "The secretome of a recombinant BCG substrain reveals differences in hypothetical proteins," Vaccine, vol. 28, no. 23, pp. 3997-4001, 2010.

[23] I. Rosenkrands, K. Weldingh, S. Jacobsen et al., "Mapping and identification of Mycobacterium tuberculosis proteins by twodimensional gel electrophoresis, microsequencing and immunodetection," Electrophoresis, vol. 21, no. 5, pp. 935-948, 2000.

[24] Y. Y. Bahk, S. A. Kim, J. S. Kim et al., "Antigens secreted from Mycobacterium tuberculosis: identification by proteomics approach and test for diagnostic marker," Proteomics, vol. 4, no. 11, pp. 3299-3307, 2004.

[25] Proteome 2D-PAGE Database, http://web.mpiib-berlin.mpg .de/cgi-bin/pdbs/2d-page/extern/index.cgi.

[26] S. Borsuk, J. Newcombe, T. A. Mendum, O. A. Dellagostin, and J. McFadden, "Identification of proteins from tuberculin purified protein derivative (PPD) by LC-MS/MS," Tuberculosis, vol. 89, no. 6, pp. 423-430, 2009.

[27] P. Orduña, M. A. Cevallos, S. P. De León et al., "Genomic and proteomic analyses of Mycobacterium bovis BCG Mexico 1931 reveal a diverse immunogenic repertoire against tuberculosis infection," BMC Genomics, vol. 12, article 493, 2011.

[28] H. Kitaura, M. Kinomoto, and T. Yamada, "Ribosomal protein L7 included in tuberculin purified protein derivative (PPD) is a major heat-resistant protein inducing strong delayed-type hypersensitivity," Scandinavian Journal of Immunology, vol. 50, no. 6, pp. 580-587, 1999.

[29] K. Kim, H. Sohn, J.-S. Kim et al., "Mycobacterium tuberculosis Rv0652 stimulates production of tumour necrosis factor and monocytes chemoattractant protein-1 in macrophages through the Toll-like receptor 4 pathway," Immunology, vol. 136, no. 2, pp. 231-240, 2012.

[30] W.-X. Xu, L. Zhang, J.-T. Mai et al., “The Wag31 protein interacts with AccA3 and coordinates cell wall lipid permeability and lipophilic drug resistance in Mycobacterium smegmatis," Biochemical and Biophysical Research Communications, vol. 448, no. 3, pp. 255-260, 2014.

[31] M. K. Gupta, V. Subramanian, and J. S. Yadav, "Immunoproteomic identification of secretory and subcellular protein antigens and functional evaluation of the secretome fraction of Mycobacterium immunogenum a newly recognized species of the Mycobacterium chelonae-Mycobacterium abscessus group," Journal of Proteome Research, vol. 8, no. 5, pp. 2319-2330, 2009.

[32] Y. Dong, S. Demaria, X. Sun et al., "HLA-A2-restricted CD8 ${ }^{+}$cytotoxic-T-cell responses to novel epitopes in Mycobacterium tuberculosis superoxide dismutase, alanine dehydrogenase, and glutamine synthetase," Infection and Immunity, vol. 72, no. 4, pp. 2412-2415, 2004.

[33] L. M. van den Berg, S. I. Gringhuis, and T. B. H. Geijtenbeek, "An evolutionary perspective on C-type lectins in infection and immunity," Annals of the New York Academy of Sciences, vol. 1253, no. 1, pp. 149-158, 2012.

[34] K. K. Huynh, S. A. Joshi, and E. J. Brown, "A delicate dance: host response to mycobacteria," Current Opinion in Immunology, vol. 23, no. 4, pp. 464-472, 2011. 


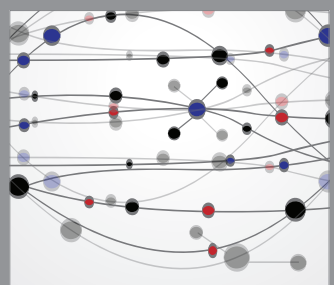

The Scientific World Journal
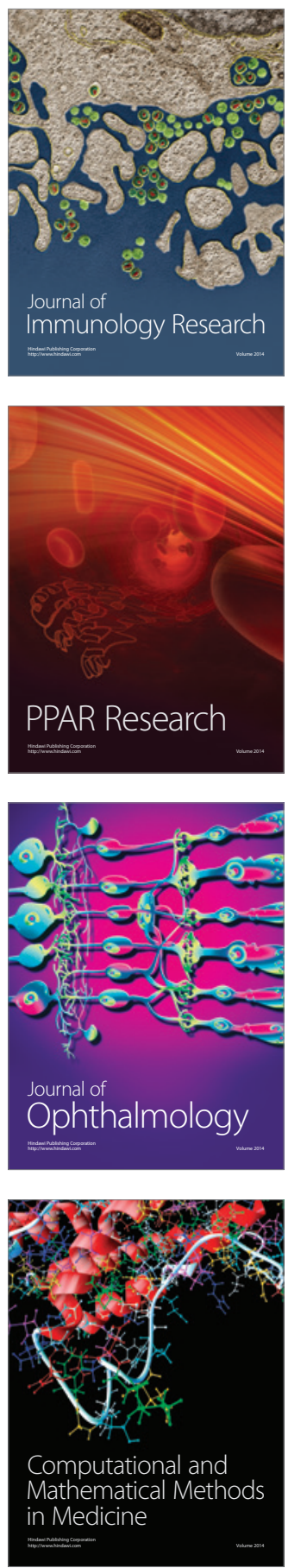

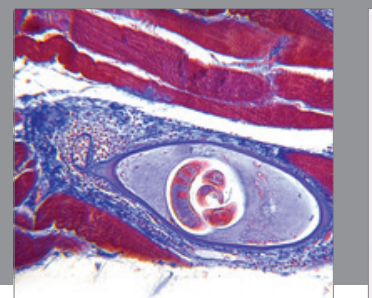

Gastroenterology

Research and Practice
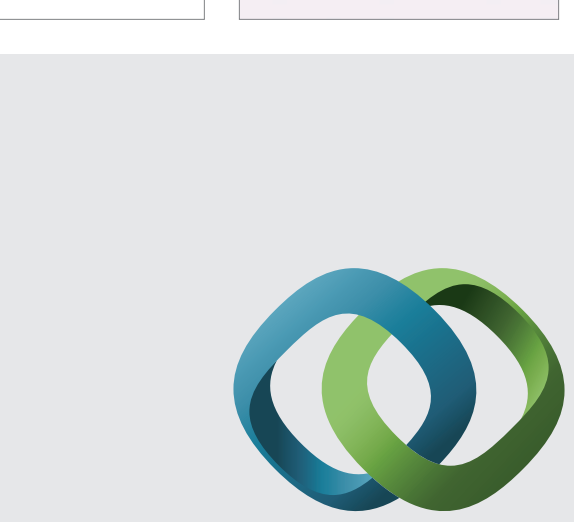

\section{Hindawi}

Submit your manuscripts at

http://www.hindawi.com
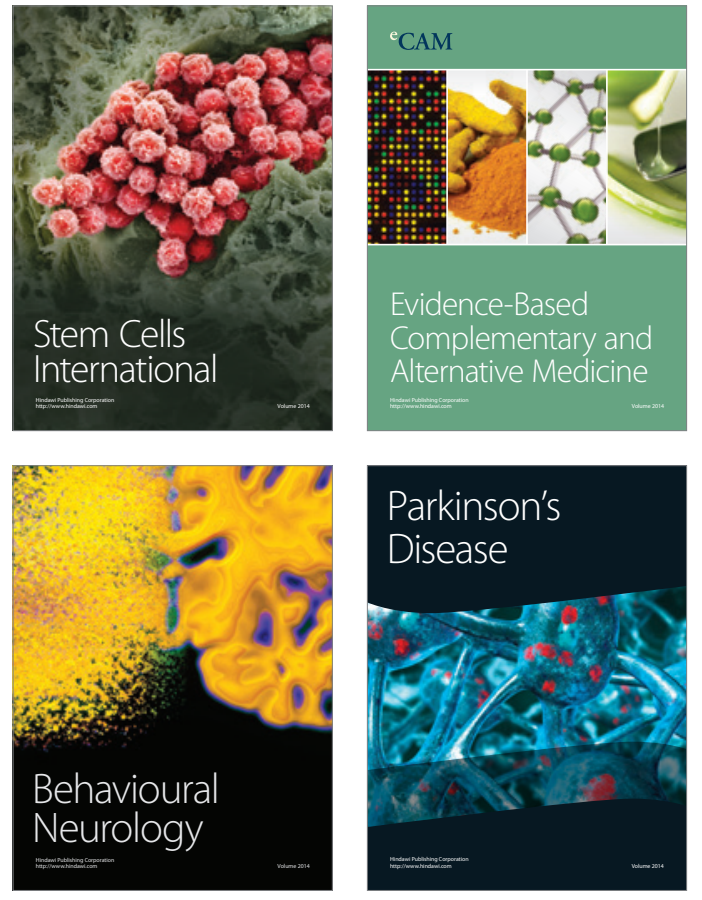
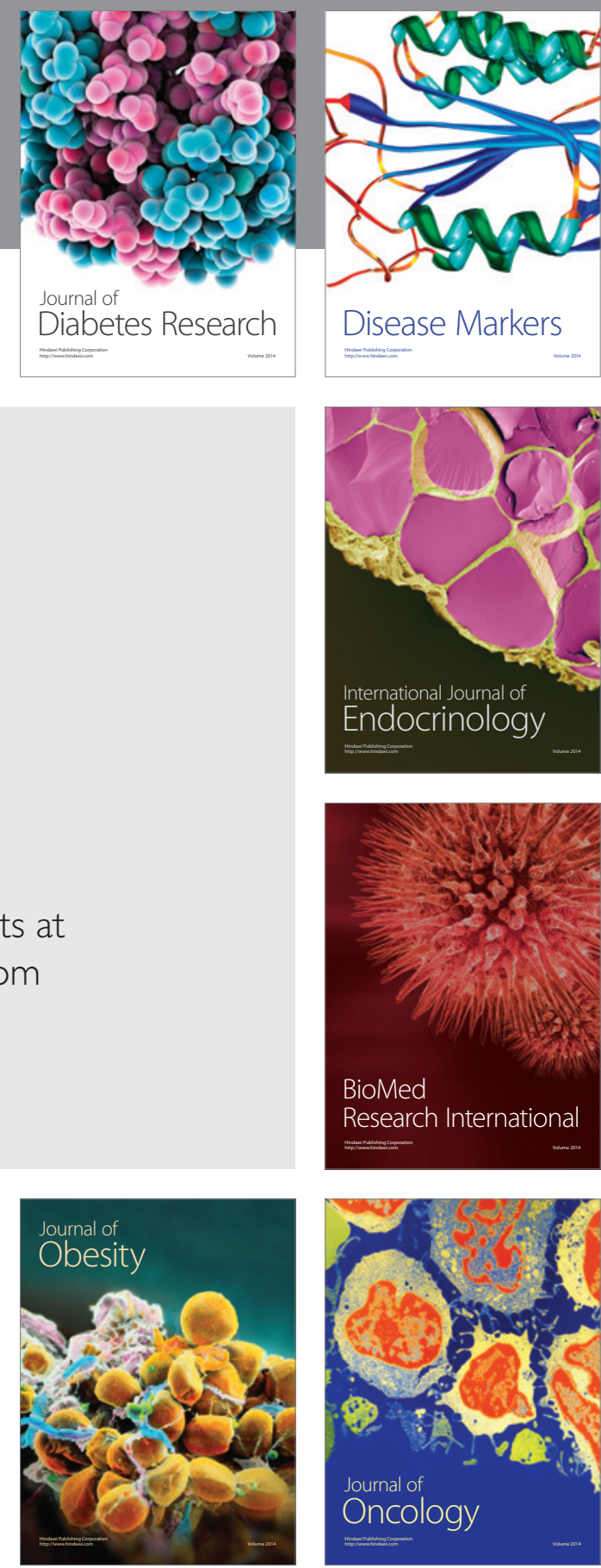

Disease Markers
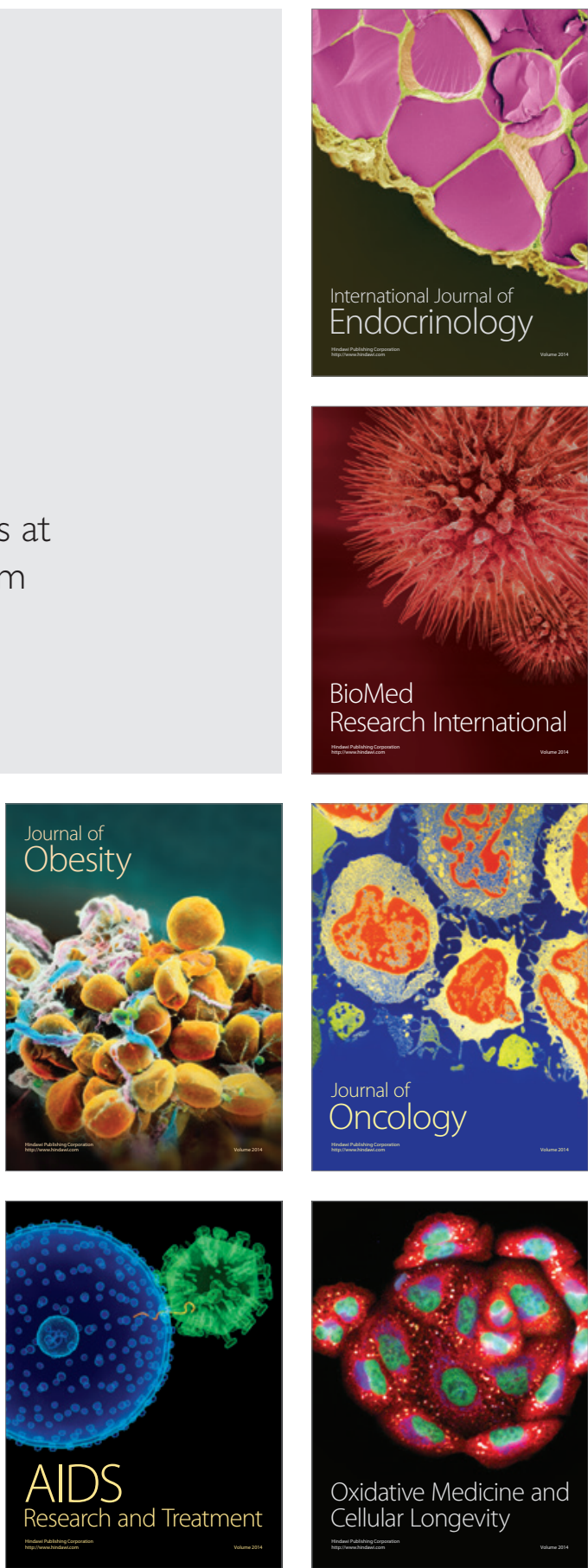